npj $\mid \begin{aligned} & \text { Systems Biology } \\ & \text { and Applications }\end{aligned}$

\title{
CORRIGENDUM OPEN
}

\section{Corrigendum: Pathway on demand: automated reconstruction of human signaling networks}

Anna Ritz, Christopher L Poirel, Allison N Tegge, Nicholas Sharp, Kelsey Simmons, Allison Powell, Shiv D Kale and TM Murali

npj Systems Biology and Applications (2016) 2, 16026; doi:10.1038/npjsba.2016.26; published online 17 November 2016

Correction to: npj Systems Biology and Applications (2016) 2, 16002; doi:10.1038/npjsba.2016.2; published online 3 March 2016

After online publication of this article, the authors noticed an error in the input files used for running the Prize Collecting Steiner Forest algorithm for Figure 2 (panels 2(a) and $2(b)$ ) and Supplementary Figure S1 in the Supplementary Information. Consequently, one sentence in the
Supplementary Information in section 2 (Algorithms for Comparison) needs to be altered.

With publication of this corrigendum, rectified Supplementary Information including corrected Supplementary Figure S1 has now been published, whereas the corrected version of Figure 2 is provided here. The authors apologize for any inconvenience caused. 
a

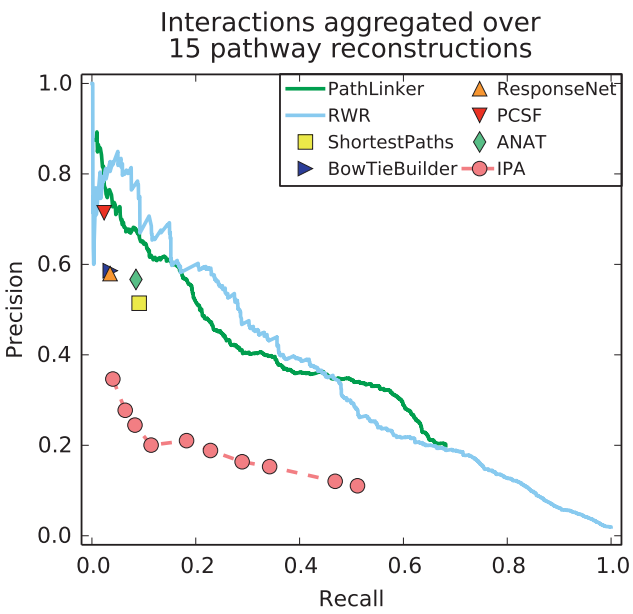

C

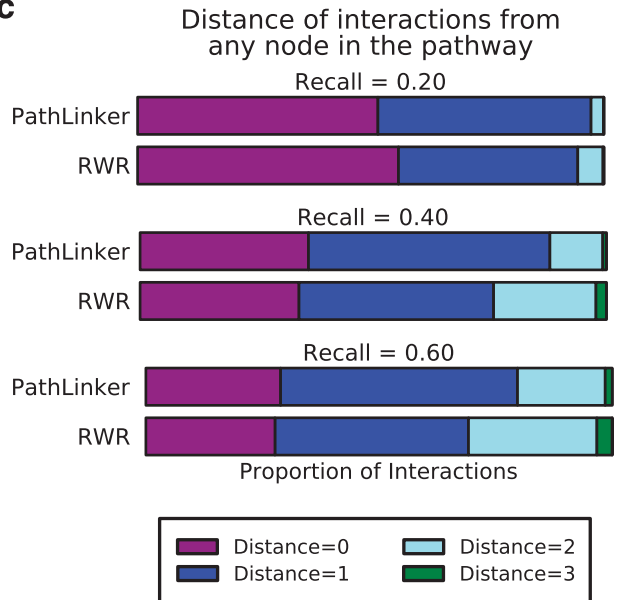

b

Interactions aggregated over

15 pathway reconstructions, ignoring pathway-adjacent negatives

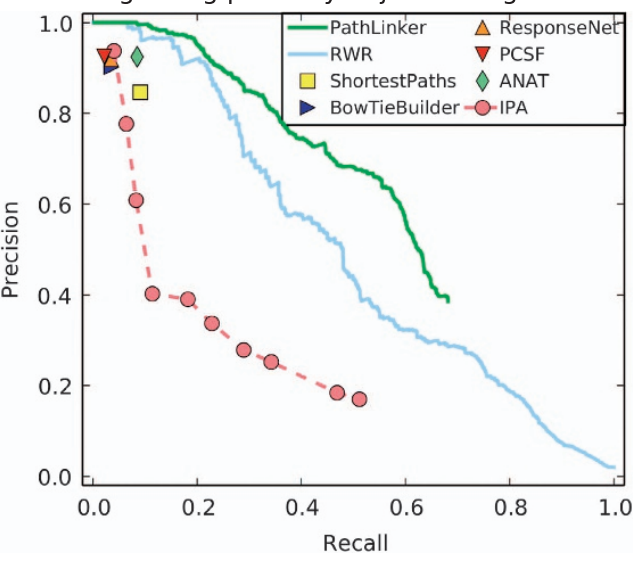

d Receptors in the aggregate reconstruction

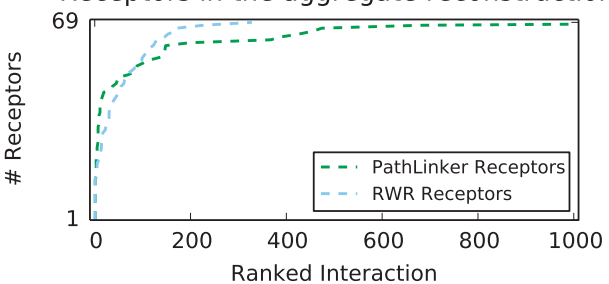

TRs in the aggregate reconstruction

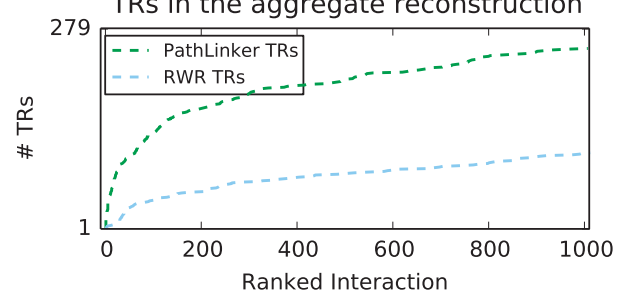

e

Additional vs. missing receptors and TRs (median over 25 random sets)

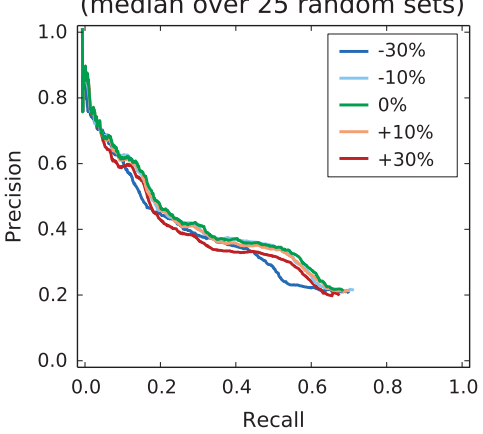

f

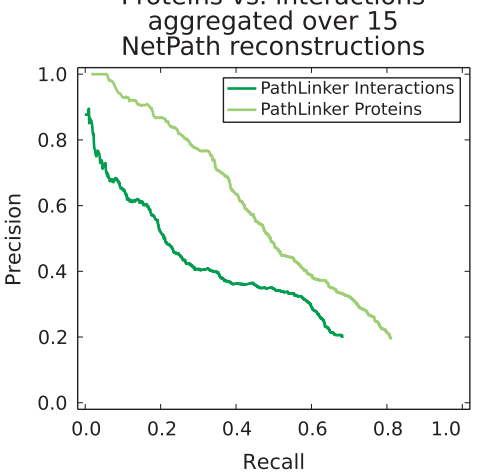

g

Interactions aggregated over Netpath and KEGG pathway databases

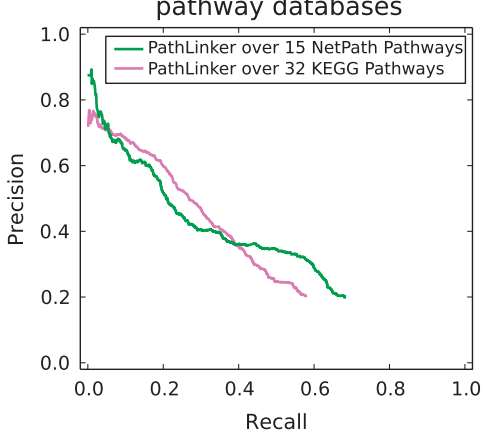

Figure 2 